\title{
A novel MRI-based finite element modeling method for calculation of myocardial ischemia effect in patients with functional mitral regurgitation.
}

1 Yue Zhang ${ }^{1,2,3}$, Vicky Y Wang ${ }^{1,2,3}$, Ashley E Morgan ${ }^{2}$, Jiwon Kim ${ }^{4}$, Liang Ge ${ }^{1,2,3}$, Julius M

2 Guccione ${ }^{1,2,3}$, Jonathan W Weinsaft ${ }^{4}$, Mark B Ratcliffe ${ }^{1,2,3^{*}}$

3 Veterans Affairs Medical Center, San Francisco, $\mathrm{CA}^{1}$,

4 Departments of Surgery ${ }^{2}$ and Bioengineering ${ }^{3}$, University of California, San Francisco, CA,

5 Department of Medicine, Weill Cornell Medicine, New York, NY4,

6 Keywords: myocardial ischemia, myocardial infarction, coronary artery disease, coronary artery

7 bypass, mitral valve insufficiency, finite element analysis, computer simulation

8 Corresponding Author: Mark Ratcliffe, Surgical Service (112), San Francisco Veterans Affairs

9 Medical Center, 4150 Clement Street, San Francisco, California 94121.

10 Telephone: (415) 221-4810 x 23730.

11 E-mail: mark.ratcliffe@ va.gov

12 Manuscript word count: 3422

13 Figure count: 8 


\section{Abstract}

15 Background: Functional Mitral Regurgitation (FMR) affects nearly 3 million patients in the United

16 States. Conventional indices are limited for predicting FMR response to coronary revascularization

17 (REVASC). Uncertainty as to which patients will respond to REVASC alone impedes rational

18 decision-making regarding FMR management. Determination of myocardial material parameters

19 associated with ischemic myocardium will address knowledge gaps regarding the impact of ischemia

20 on regional cardiac muscle.

21 Method: We proposed a novel MRI-based finite element (FE) modeling method to determine the

22 effect of ischemia on myocardial contractility. The method was applied to two patients with multi-

23 vessel coronary disease and FMR and one healthy volunteer. Cardiac MRI (CMR) included cine-

24 MRI, gadolinium-enhanced stress perfusion, late gadolinium enhancement (LGE), and non-invasive

25 tagged MRI (CSPAMM). The left ventricular (LV) FE model was divided into 17 sectors. Sector-

26 specific circumferential and longitudinal end-systolic strain and LV volume from CSPAMM were

27 used in a formal optimization to determine the sector based myocardial contractility, $\mathrm{T}_{\max }$.

28 Results: The FE optimization successfully converged with good agreement between calculated and

29 experimental end-systolic strain and LV volumes. Specifically, the optimized Tmax $\operatorname{mor}_{H}$ Patient 1,

30 Patient 2, and the volunteer was $336.8 \mathrm{kPa}, 401.4 \mathrm{kPa}$, and $259.4 \mathrm{kPa}$ and $\alpha$ for Patient 1 and Patient

312 was 0.44 and 0.

32 Conclusion: We developed a novel computational method able to predict the effect of myocardial

33 ischemia in patients with FMR. This method can be used to predict the effect of ischemia on the

34 regional myocardium and promises to facilitate better understanding of FMR response to REVASC.

35 Abstract Word count: 249 (limit 350) 


\section{Introduction}

38 Functional mitral regurgitation (FMR) is a leading cause of valvular heart disease. FMR occurs in

39 nearly 3 million people in the United States, more than 400,000 of whom have advanced $(\geq$

40 moderate) MR (Gorman et al., 2003; Groarke et al., 2017). Left ventricular ischemia is a potential

41 causal factor for FMR, as evidenced by animal studies showing that ischemia induction via coronary

42 ligation yields acute MR which resolves after restoration of coronary patency (Kono et al., 1992;

43 Messas et al., 2001). Although these data support the notion that FMR can be effectively treated

44 when ischemia is relieved, clinical uncertainty persists as to whether ischemia is a primary

45 determinant of FMR response to coronary revascularization in the context of confounding variables

46 such as myocardial infarction (MI) and left ventricular (LV) chamber remodeling. As evidence of

47 this, clinical studies have shown that in approximately half of patients, MR improves with coronary

48 revascularization (REVASC), whereas in the remainder MR persists or worsen (Aklog et al., 2001;

49 Penicka et al., 2009; Kang et al., 2011). Uncertainty as to which patients will respond to

50 revascularization impedes rational decision-making regarding MR management.

51 Finite element (FE) modeling has the unique potential to discern the impact of ischemia on MR in

52 context of concomitant LV remodeling and infarction. Prior studies by our group (Walker et al.,

53 2005; Wenk et al., 2011) and others (Bogen et al., 1980; Genet et al., 2015) have focused on the

54 impact of MI alone on FMR but without incorporation of ischemia effect into computational models.

55 Other FE modeling studies have incorporated ischemia into simulations focused on arrhythmogenesis

56 (Wang et al., 2013; Mendonca Costa et al., 2018) without incorporation of LV geometry or function.

57 McCulloch described the implementation of the coronary blood flow myocardial function

58 relationship in a FE framework (McCulloch and Mazhari, 2001). However, to date, modeling of

59 ischemia effect on LV contractility as occurs in infarcted and hibernating myocardium (Wijns et al.,

60 1998) has not been described in the context of advanced LV remodeling with multivessel coronary

61 disease (CAD).

62 New advances in non-invasive imaging enable both LV ischemia and infarction to be assessed with

63 high precision, facilitating inclusion of such data into FE models. Cardiac magnetic resonance

64 (CMR) has been well validated for both LV remodeling and tissue characterization. Cine-CMR has

65 been employed as a reference standard for LV chamber size and function (von Knobelsdorff-

66 Brenkenhoff et al., 2017). Stress perfusion (SP) CMR has been shown to provide high diagnostic

67 accuracy for ischemia as can occur with obstructive CAD (Bruder et al., 2013). Late gadolinium

68 enhancement (LGE) CMR provides near exact agreement with histopathology evidenced myocardial 
69 infarction (Kim et al., 1999; Kim et al., 2000) and CMR with non-invasive tags (CSPAMM) has been

70 widely used in assessing LV contractility (Ryf et al., 2002). This study leveraged multiparametric

71 CMR data to develop and validate a novel computational method to predict the effect of myocardial 72 ischemia on regional left ventricular function in patients with FMR. 


\section{Methods}

75 Two patients and one healthy volunteer were prospectively enrolled in a protocol examining FMR76 associated remodeling. Imaging was performed at Weill Cornell Medical College (New York, NY)

77 using 3.0 Tesla scanners (General Electric, Waukesha, WI) (Morgan et al., 2018). The Cornell

78 Institutional Review Board approved this study, and written informed consent was obtained at time

79 of enrollment.

\section{$80 \quad 2.1 \quad$ Computational modeling pipeline}

81 The proposed method is summarized in Figure 1. The CMR images were used to create the LV \& 82 right ventricular (RV) surfaces (Section 2.3), to calculate the 3D regional strains (Section 2.6), and

83 to configure the LV mechanical parameters (Section 2.7). Along with the patient-specific LV and RV 84 pressures, the LV mechanics models were constructed using the LV and RV surfaces, rule-based

85 fiber angles, diastolic and systolic myocardial constitutive relationships and LV myocardial material

86 parameters. A formal parameter optimization framework was then constructed based on the LV

87 mechanics models and the in vivo sector-specific circumferential and longitudinal end-systolic strain 88 and LV volume.

\subsection{Image acquisition}

90 Four CMR sequences were collected for each subject. The ventricular structure/function were

91 assessed by Cine CMR with steady-state free precession (Figure 2A). The LV ischemia was assessed

92 by the Gadolinium-enhanced first pass Regadenoson-induced SP (4-5 equidistant LV short-axis

93 images) (Figure 2B). The LV infarction was assessed by the delayed-enhancement inversion

94 recovery CMR for LGE, $10-30$ minutes after administration of gadolinium $(0.2 \mathrm{mmol} / \mathrm{kg}) \mathrm{using}$ a

95 segmented inversion recovery sequence, with inversion time tailored to null viable myocardium

96 (Figure 2C). CSPAMM in contiguous LV short and long axis slices ( $8 \mathrm{~mm}$ tag spacing, $10 \mathrm{~mm}$ slice

97 thickness, no gap) were performed to measure the myocardial deformation and strain (Figure 2D).

\section{$\begin{array}{lll}98 & \mathbf{2 . 3} & \text { Image analysis }\end{array}$}

\subsubsection{LV and RV contouring}

100 The LV (both epicardium and endocardium) short and long axis and the RV (only endocardium)

101 short axis Cine-CMR were contoured at LV early diastolic filling phase (EDF) in a medical image

102 processing platform, MeVisLab (version 2.7.1, Bremen, DE). The EDF phase was defined as the 
103 beginning of the opening of the mitral valve. The short axis Cine-CMR were contoured from apex to

104 the mitral valve plane. For this study, only short-axis contours were used for surface generation. The

$105 \mathrm{LV}$ and RV contours included the papillary muscle bases but excluded the trabeculae in this study.

106 An example is shown in Figure 2A.

107 In plane shift of the short axis cine-CMR due to breathing resulted in a misalignment of the LV and

108 RV short axis contours. To eliminate this in-plane misalignment, the LV epicardium in three LV long 109 axis cine-CMR (2-, 3- and 4-chamber views) were contoured. A LV epicardial surface was created

110 based on those three long axis contours and this 3D surface had a cross section in each short axis

111 image plane. The centroid of each cross section was calculated and denoted as $\boldsymbol{A}$. Each LV short axis

112 epicardial contour had a calculated centroid denoted as $\boldsymbol{A}^{\prime}$. A transform vector for each short axis

113 contour then was calculated using $\boldsymbol{A}-\boldsymbol{A}^{\prime}$. This transform vector for each image plane was applied on

114 both LV and RV short axis epicardial and endocardial contours. After the alignment, the LV

115 epicardial and endocardial and RV endocardial surfaces were created using the aligned short axis

116 contours.

\section{$117 \quad$ 2.3.2 MRI-measured Strains}

118 Regional 3D circumferential and longitudinal strains for each sector were calculated from CSPAMM 119 images using an in-house software as described previously (Morgan et al., 2018). The HARmonic

120 Phase (HARP) method developed by Osman et al. (Osman et al., 2000) was implemented. The

121 implementation details has been reported by Morgan et al. in the Appendix of (Morgan et al., 2018).

\section{$122 \quad 2.4 \quad$ LV FE model generation}

123 The LV epicardial and endocardial surfaces were input to TruGrid (XYZ Scientific Applications Inc,

124 Pleasant Hill, California, USA) to mesh the LV as shown in Figure 3. Meshes with different density

125 were created. Optimal mesh density was determined as a function of parameter calculation accuracy

126 and calculation time. Custom software (C\#, Visual Studio 2017, Microsoft, Redmond, WA) that

127 included a ray casting method (Möller and Trumbore, 1997) to locate the interventricular septum

128 (Figure 3A) was used to break the mesh into 17 AHA sectors (Cerqueira et al., 2002) (Figure 3B).

\section{$129 \quad 2.5 \quad$ Loading and boundary condition}

130 The LV systolic pressure was obtained from blood pressure cuff and LV ED and RV ED and ES

131 pressures were estimated from concomitant transthoracic echocardiographic examination data

132 (Ommen et al., 2000). Pressure data is summarized in Table 1. Patient-specific LV and RV pressures 
133 were applied on the LV endocardial and LV septal epicardial surfaces, respectively. Nodes at the LV

134 top surface were applied with homogeneous Dirichlet boundary conditions. Briefly, basal enpicardial

135 and endocardial nodes were able to slide within the LV valve plane during passive filling and systolic

136 contraction.

\section{$137 \quad 2.6 \quad$ Constitutive laws}

138 Passive and active myocardial constitutive laws described by Guccione et al. (Guccione et al., 1991;

139 Guccione et al., 1993) were used in this study. The LV myocardium was modeled with a user-defined

140 material subroutine in the explicit FE solver, LS-DYNA (Liver- more Software Technology

141 Corporation, Livermore, CA). Each FE model was assigned with the rule-based fiber angles with

142 myofiber helix angle varying transmurally from $60^{\circ}$ at the endocardium to $-60^{\circ}$ at the epicardium.

\section{$143 \quad 2.7 \quad$ Sector-based myocardial material parameters}

144 The LGE and SP scores were defined as integers from 0 to 4, where 0 represents healthy and 4 the most severe. Passive stiffness, $C$, was determined using the following:

$$
C_{n}=C_{H}+9 \cdot C_{H} \cdot \frac{L G E_{n}}{4}
$$

where $C_{n}$ represents the passive stiffness for each sector and $C_{H}$ the stiffness of a 'healthy' sector with

$148 \mathrm{LGE}=0$. Note that when LGE $=4$ (transmural MI), $C_{n}=10 C_{H}$ which is consistent with our prior 149 work (Wenk et al., 2011).

150 On the other hand, both LGE and SP were assumed to effect regional contractility, Tmax, according to the following linear relationship:

$$
\operatorname{Tmax}_{n}=\operatorname{Tmax}_{H} \cdot\left(1-\frac{L G E_{n}}{4}\right) \cdot\left(1-\alpha \cdot \frac{S P_{n}}{4}\right),
$$

153 where $\operatorname{Tmax}_{n}$ represents the contractility for each sector, $\operatorname{Tmax}_{H}$ the contractility for healthy sector 154 with $\mathrm{LGE}$ and $\mathrm{SP}=0, \alpha$ the ischemia effect, and $L G E_{n}$ and $S P_{n}$ the LGE and SP scores for each sector. 155 It's known that $\operatorname{Tmax}_{n} \geq 0$ and $L G E_{n}$ and $S P_{n}$ are $\ni[0,4]$, then it can be determined $\alpha \ni[0,1]$. Since

156 we assumed that the LGE and SP scores were 0 for the healthy volunteer, $C_{n}$ and $\operatorname{Tmax}_{n}$ for the 157 volunteer were equal to $C_{H}$ and $\operatorname{Tmax}_{H}$, respectively.

\section{$158 \quad 2.8 \quad$ Model optimization}

159 A formal optimization of $C_{H}, \operatorname{Tmax}_{H}$, and $\alpha$ were performed where the objective function for the 160 optimization was taken to be the mean-squared errors (MSE) (Guccione et al., 2001). $C_{H}$ was 
161 determined such that the FE model predicted LV end-diastolic volume matched the patient-specific in

162 vivo measured volume. $\operatorname{Tmax}_{H}$ was estimated by minimizing the MSE between FE model-predicted

163 and in vivo MRI-measured end-systolic longitudinal and circumferential strains and the LV end-

164 systolic volume. The goal of the optimization is to minimize the MSE as follows:

165 Objective function $=\frac{1}{2 N}\left(\sum_{n=1}^{N}\left(\left(E_{c c, n}-\bar{E}_{c c, n}\right)^{2}+\left(E_{l l, n}-\bar{E}_{l l, n}\right)^{2}\right)\right)+W\left(\frac{V_{E S}-\bar{V}_{E S}}{\bar{V}_{E S}}\right)^{2}$,

166 where $n$ is the in vivo average strain at each sector (note the apex sector was excluded so $n=16$ ), $E_{c c, n}$

167 the calculated FE circumferential strain, $E_{l l, n}$ the calculated longitudinal strain, $V_{E S}$ the LV end-

168 systolic volume and $W$ is the weight applied to the volume term. The overbar represents the 169 experimental in vivo measurements.

$170 W=10$ was applied to the volume term to make the strain and volume effects more balanced. For each

171 case, the $\operatorname{Tmax}_{H}$ was initially $=350 \mathrm{kPa}$. $\alpha$ was initially $=0.5$ in the FMR patient models and $=0$ in

172 the healthy volunteer model. No constraints were applied to either $\operatorname{Tmax}_{H}$ or $V_{E S}$. 


\section{Results}

175 Patient specific LV and RV pressures are shown in Table 1 and patient specific LGE, SP and wall 176 motion scores are shown in Table 2.

177 Briefly, Patient 1 was an 88 year old man with multi-vessel CAD (prior left main coronary

178 percutaneous coronary intervention (PCI) with residual chronic occlusion of the left circumflex

179 coronary), heart failure, and severe FMR. CMR demonstrated an enlarged LV with severely reduced

180 LV systolic function (ejection fraction (EF) 30\%), subendocardial MI with moderate-severe inducible

181 ischemia involving the inferior and lateral walls as well as subendocardial MI with mild-moderate

182 inducible ischemia involving the LV anterior wall.

183 Patient 2 was an 84 year old woman with multi-vessel CAD (prior multi-vessel PCI), heart failure,

184 moderate MR. Cardiac MRI demonstrated normal LV size with mildly reduced LV systolic function

185 (EF 45\%), transmural inferolateral MI, and mild-moderate inducible ischemia involving the LV

186 anterior wall.

187 The healthy volunteer had no known heart disease. Note that wall motion was normal and LGE and

188 SP were assumed $=0$ for the healthy volunteer.

\section{$189 \quad 3.1 \quad$ Mesh convergence study}

190 A mesh convergence study was performed on Patient 1 and Patient 2 to find the minimum number of 191 elements needed to obtain stable calculations of $\mathrm{C}_{H}, \operatorname{Tmax}_{H}$, and $\alpha$ within the fastest computation

192 time. 4 sets of meshes with different density were created with the transmural elements from 3 to 4 ,

193 circumferential elements from 40 to 64, and longitudinal elements from 22 to 38 . This study

194 determined that 7952 elements (Table 3) are required and further mesh refinement only results in an

195 average of $2 \%$ change in $C,<2 \%$ change in $\operatorname{Tmax}_{H}$ and no change in $\alpha$. Note that only the

196 optimization results for using 7952 elements will be presented in the following sections.

\section{$197 \quad 3.2 \quad$ Testing with synthetic data}

198 Method accuracy was determined using idealized input data. Briefly, two simulations of diastolic inflation and systolic contraction for each patient were firstly conducted by setting $\alpha$ to be 0 and 1 ,

200 respectively (note that $C_{H}$ and $\operatorname{Tmax}_{H}$ were set as the optimized values as presented in Section 3.2).

201 For each case, the simulated strain and LV ESV were used as input in our optimization framework as

202 "experimental data". For each case, the optimization was initiated with $\alpha$ initially set at 0.5 and 
$203 \operatorname{Tmax}_{H} 350 \mathrm{kPa}$. Figure 4 shows excellent agreement between the synthetically generated

204 “experimental strain" and the strain after optimization. The target and optimized parameters are

205 summarized in Table 4 with a maximal difference of 1.4\%, which shows the proposed method has

206 great capability of predicting $\operatorname{Tmax}_{H}$ and $\alpha$ accurately.

\subsection{Prediction of $\mathrm{C}_{H}, \operatorname{Tmax}_{H}$, and $\alpha$}

$208 C_{H}$ was determined such that the FE model predicted LV EDV matched the patient-specific in vivo 209 measured EDV (Table 5).

210 Figure 5A shows excellent convergence of the objective function (OF) during a representative model

211 optimization. Figure 5B shows a surface plot of MSE of the strain respect to in $\operatorname{Tmax}_{H}$ and $\alpha$, where

212 the valley of the surface indicates lowest MSE value relating to the best match between the

213 experimental and FE model-predicted strain. The optimized $\operatorname{Tmax}_{H}$ for Patient 1, Patient 2, and the

214 volunteer was $336.8 \mathrm{kPa}, 401.4 \mathrm{kPa}$, and $259.4 \mathrm{kPa}$ and $\alpha$ for Patient 1 and Patient 2 was 0.44 and 0.

215 The optimized $\operatorname{Tmax}_{H}$ and $\alpha$ were obtained with the displaying good convergence as shown in

216 Figure 6. The final OF value of 0.45 (an average calculated using all three cases) was obtained

217 indicating generally good agreement between the FE model-predicted systolic strain and the patient-

218 specific in vivo measured strain. The LV ESV was accurately predicted at $108.3 \mathrm{ml}, 51.3 \mathrm{ml}$, and

$21948.6 \mathrm{ml}$, respectively for Patient 1, Patient 2, and volunteer, which was averagely only $2 \%$ smaller

220 than the volume measured from MRI data.

221 Figure 7A shows a comparison of the average of $E_{c c}$ across all 16 sectors between the experimental

222 data and calculated with the FE model. In general, FE model predicted strain agreed with

223 experimental data although $E_{c c}$ was slightly underestimated in Patients 12 and slightly overestimated

224 in the volunteer. Figure 7B shows that, for Patient 1 and Patient 2, the difference of the average of

$225 E_{c c}$ between the experimental data and the FE model prediction is significantly reduced when only

226 including the sectors with LGE score $<3$.

\section{$227 \quad 3.4 \quad$ Virtual REVASC}

228 Given that the FE model predicted a non-zero ischemic effect for Patient 1 (e.g. alpha = 0.44), a

229 virtual REVASC simulation was performed by running a simulation with $\alpha$ reduced to 0 while

230 keeping other parameters unchanged. The comparison of the average of $E_{c c}$ between baseline and

231 post-REVASC is shown in Figure 8, where it can be found that the virtual REVASC improved 
232 Patient 1's $E_{c c}$ by $10.7 \%$. The LV ESV after REVASC was $102 \mathrm{ml}$, which was $6 \%$ smaller than that 233 at baseline. 


\section{Discussion}

235 In this study, we demonstrated a novel method to estimate the effect of ischemia on regional LV

236 myocardial contractility. The proposed method takes the advantage of multiple CMR sequences and

237 the FE modeling to formally optimize the LV mechanics parameters. A linear relationship was

238 proposed to describe the effect of LV infarct and ischemia on LV active contraction. Our approach

239 was tested with CMR data from two patients with multi-vessel coronary disease and moderate FMR

240 and a healthy volunteer. Good agreement between the FE model-predicted systolic strains and the

241 patient-specific in vivo measured strains were observed, which suggests that the optimized model was

242 faithful to the experimental data.

\section{$243 \quad 4.1 \quad$ Ischemia effect}

244 The effect of ischemia, $\alpha$, on LV contractility was found to be 0.44 for Patient 1 while 0 for Patient 2.

245 We suggest that this is consistent with the patient specific perfusion data seen in Table 2 where the

246 cumulative SP score of Patient 1 in sectors with LGE > 3 is 19 while the cumulative ischemia in

$247 \quad$ Patient 2 is only 4.

248 It should be noted that stress induced perfusion defects are associated with either normal or depressed

249 regional myocardial function and in the latter case the myocardium would be described as

250 hibernating (Wijns et al., 1998). First, by definition, our method identifies only hibernating

251 myocardium and simple demand ischemia without dysfunction is not considered. As a corollary, if a

252 patient with multi-vessel CAD had a large amount of demand ischemia without LV dysfunction, our

253 method would not identify an ischemia effect. Specifically, if the SP defect is showing simple

254 demand ischemia without underlying contractility deficit, then $\alpha$ would likely be 0 . If the SP defect is

255 associated with hibernating myocardium where contractility is reduced, then $\alpha$ will likely be $>0$.

\subsection{MI stiffness}

257 Our assumption that infarct stiffness is ten times that of normal myocardium is consistent with our

258 prior studies (Walker et al., 2005; Wenk et al., 2011) and others (Genet et al., 2015), there is reason

259 to believe that better measurement of infarct stiffness is necessary in patients with multi-vessel

260 coronary disease and FMR. First, as seen in Figure 7B, model accuracy was much better when

261 sectors with transmural infarction were excluded. Second, the optimized $\operatorname{Tmax}_{H}$ was higher in the

262 two patients with multi-vessel coronary (336.8 and $401.4 \mathrm{kPa}$ respectively) than the healthy volunteer

$263(259.4 \mathrm{kPa})$. The reason for this discrepancy is unclear but might be explained if Equation 2 is 
overestimating the effect of infarct and ischemia on LV contractility. It is anticipated that future studies that measure infarct stiffness in patients with MI but without ischemia will help to better determine the relationship between infarct and myocardial contractility.

\subsection{Diastolic stiffness in patients with multi-vessel CAD}

268 As seen in Table 3, there is a large variance in optimized $C_{H}$ between the healthy volunteer and 269 patients with multi-vessel CAD and FMR. We suspect this is likely a function of myocardial fibrosis

270 (LGE scores > 0) and, consistent with this, prior clinical studies have shown the extent of LGE to 271 correspond to impaired LV relaxation (Moreo et al., 2009). However, the cumulative LGE scores of

272 Patients 1 and 2 are similar and further studies are therefore necessary to establish our hypothesis. On

273 the other hand, the lack of diastolic strain in this study limits our ability to better optimize passive LV 274 material parameters.

\section{$275 \quad 4.4$ Model accuracy}

276 Results of a mesh sensitivity study suggest that the optimized LV material parameters are relatively 277 insensitive to the mesh density, although slight differences were observed between FE models with 278 different mesh density. Table 2 indicates that a mesh with 7952 may be the optimal choice for the 279 current study because of the balanced calculation time and parameter optimization accuracy.

280 Furthermore, synthetic test results suggest that the proposed method is capable of precisely

281 determining the LV passive and active material properties and the ischemia effect on LV active 282 contractility.

\subsection{Virtual revascularization}

284 As listed in Table 2, sectors 5 and 11 in Patient 1 had SP score equal to 3. However, expected strain 285 improvement was not found in those sectors after a virtual REVASC. A possible explanation is that 286 sectors 5 and 11 had LGE score equal to 3, which indicated for those sectors, the reduction in 287 contractility was dominated by the LV myocardial infarct. This suggests that for LV myocardium 288 with severe infarction, the benefit from restoring the blood supply is limited.

\subsection{Limitations}

290 First, only systolic strains were calculated since the CSPAMM data were acquired during the systolic 291 phase, which resulted in a fact that only systolic myocardial material parameters can be optimized. 
292 Because of the lack of diastolic strains, the main diastolic material parameter, $C_{H}$, was calibrated to

293 match the measured LV end-diastolic volume. Second, the border zone effect was not taken into

294 consideration in the current study. Third, only a linear relationship between the infarct and ischemia

295 and the LV contractility were employed in this study. Fourth, the LV base boundary conditions may

296 affect the FE-model-based longitudinal strain calculation. 


\section{Conclusion}

298 This study proposed a novel and efficient method to predict the effect of ischemia on the LV

299 contractility with the help of multiple CMR acquisitions and the FE modeling. The proposed method

300 has good agreement between the FE model-predicted systolic strains and the patient-specific in vivo

301 measured strains. The proposed method may hold the basis for a better understanding of FMR

302 response to REVASC. 


\section{Acknowledgments}

305 This study was supported by NIH grants R01-HL63348/Ratcliffe and R01-HL128278/Weinsaft. This

306 support is appreciated. 


\section{Author Contributions Statement}

309 YZ, JW and MR designed the study. YZ conducted simulations, analyzed results, and wrote the

310 initial draft of the paper. MR created the finite element models. VW, AM, and JK did data analysis.

311 LG contributed to development of methodology. JW collected the clinical data. LG, JW, and MR

312 supervised the study. YZ, VW, JG, JW and MR contributed to manuscript writing/review. 


\section{Conflict of Interest Statement}

315 The authors declare that the research was conducted in the absence of any commercial or financial

316 relationships that could be construed as a potential conflict of interest. 
Aklog, L., Filsoufi, F., Flores, K.Q., Chen, R.H., Cohn, L.H., Nathan, N.S., et al. (2001). Does coronary artery bypass grafting alone correct moderate ischemic mitral regurgitation? Circulation 104(12 Suppl 1), I68-75.

Bogen, D.K., Rabinowitz, S.A., Needleman, A., McMahon, T.A., and Abelmann, W.H. (1980). An analysis of the mechanical disadvantage of myocardial infarction in the canine left ventricle. Circ Res 47(5), 728-741.

Bruder, O., Wagner, A., Lombardi, M., Schwitter, J., van Rossum, A., Pilz, G., et al. (2013). European Cardiovascular Magnetic Resonance (EuroCMR) registry--multi national results from 57 centers in 15 countries. J Cardiovasc Magn Reson 15, 9. doi: 10.1186/1532-429X$15-9$.

Cerqueira, M.D., Weissman, N.J., Dilsizian, V., Jacobs, A.K., Kaul, S., Laskey, W.K., et al. (2002). Standardized myocardial segmentation and nomenclature for tomographic imaging of the heart. A statement for healthcare professionals from the Cardiac Imaging Committee of the Council on Clinical Cardiology of the American Heart Association. Circulation 105(4), 539542.

Genet, M., Chuan Lee, L., Ge, L., Acevedo-Bolton, G., Jeung, N., Martin, A., et al. (2015). A Novel Method for Quantifying Smooth Regional Variations in Myocardial Contractility Within an Infarcted Human Left Ventricle Based on Delay-Enhanced Magnetic Resonance Imaging. J Biomech Eng 137(8), 081009. doi: 10.1115/1.4030667.

Gorman, R.C., Gorman, J.H., 3rd, and Edmunds, L.H., Jr (2003). "Ischemic mitral regurgitation in cardiac surgery in the adult," in Cardiac Surgery in the adult, eds. L.H. Cohn \& L.H. Edmunds, Jr. (New York: McGraw-Hill), 1751-1770.

Groarke, J., Carabello, B., O’Gara, P., Fuster, V., Harrington, R., Narula, J., et al. (2017). "Ischemic Mitral Regurgitation," in Hurst's The Heart,. (New York, NY: McGraw-Hill Education).

Guccione, J.M., McCulloch, A.D., and Waldman, L.K. (1991). Passive material properties of intact ventricular myocardium determined from a cylindrical model. J Biomech Eng 113(1), 42-55.

Guccione, J.M., Moonly, S.M., Moustakidis, P., Costa, K.D., Moulton, M.J., Ratcliffe, M.B., et al. (2001). Mechanism underlying mechanical dysfunction in the border zone of left ventricular aneurysm: a finite element model study. Ann Thorac Surg 71(2), 654-662.

Guccione, J.M., Waldman, L.K., and McCulloch, A.D. (1993). Mechanics of active contraction in cardiac muscle: Part II--Cylindrical models of the systolic left ventricle. J Biomech Eng 115(1), 82-90.

Kang, D.H., Sun, B.J., Kim, D.H., Yun, S.C., Song, J.M., Choo, S.J., et al. (2011). Percutaneous versus surgical revascularization in patients with ischemic mitral regurgitation. Circulation 124(11 Suppl), S156-162. doi: 10.1161/CIRCULATIONAHA.110.011254.

Kim, R.J., Fieno, D.S., Parrish, T.B., Harris, K., Chen, E.L., Simonetti, O., et al. (1999). Relationship of MRI delayed contrast enhancement to irreversible injury, infarct age, and contractile function. Circulation 100(19), 1992-2002.

Kim, R.J., Wu, E., Rafael, A., Chen, E.L., Parker, M.A., Simonetti, O., et al. (2000). The use of contrast-enhanced magnetic resonance imaging to identify reversible myocardial dysfunction. N Engl J Med 343(20), 1445-1453. doi: 10.1056/NEJM200011163432003.

Kono, T., Sabbah, H.N., Rosman, H., Alam, M., Jafri, S., Stein, P.D., et al. (1992). Mechanism of functional mitral regurgitation during acute myocardial ischemia. J Am Coll Cardiol 19(5), 1101-1105.

McCulloch, A.D., and Mazhari, R. (2001). Regional myocardial mechanics: integrative computational models of flow-function relations. J Nucl Cardiol 8(4), 506-519. doi: 10.1067/mnc.2001.117113. 
366

367

368

369

370

371

372

373

374

375

376

377

378

379

380

381

382

383

384

385

386

387

388

389

390

391

392

393

394

395

396

397

398

399

400

401

402

403

404

405

406

407

408

409
Mendonca Costa, C., Plank, G., Rinaldi, C.A., Niederer, S.A., and Bishop, M.J. (2018). Modeling the Electrophysiological Properties of the Infarct Border Zone. Front Physiol 9, 356. doi: 10.3389/fphys.2018.00356.

Messas, E., Guerrero, J.L., Handschumacher, M.D., Chow, C.M., Sullivan, S., Schwammenthal, E., et al. (2001). Paradoxic decrease in ischemic mitral regurgitation with papillary muscle dysfunction: insights from three-dimensional and contrast echocardiography with strain rate measurement. Circulation 104(16), 1952-1957.

Möller, T., and Trumbore, B. (1997). Fast, Minimum Storage Ray-Triangle Intersection. Journal of Graphics Tools 2(1), 21-28. doi: 10.1080/10867651.1997.10487468.

Moreo, A., Ambrosio, G., De Chiara, B., Pu, M., Tran, T., Mauri, F., et al. (2009). Influence of myocardial fibrosis on left ventricular diastolic function: noninvasive assessment by cardiac magnetic resonance and echo. Circ Cardiovasc Imaging 2(6), 437-443. doi: 10.1161/CIRCIMAGING.108.838367.

Morgan, A.E., Zhang, Y., Tartibi, M., Goldburg, S., Kim, J.J., Nguyen, T.D., et al. (2018). Ischemic Mitral Regurgitation: Abnormal Strain Overestimates Nonviable Myocardium. Ann Thorac Surg 105(6), 1754-1761. doi: 10.1016/j.athoracsur.2018.01.005.

Ommen, S.R., Nishimura, R.A., Appleton, C.P., Miller, F.A., Oh, J.K., Redfield, M.M., et al. (2000). Clinical utility of Doppler echocardiography and tissue Doppler imaging in the estimation of left ventricular filling pressures: A comparative simultaneous Doppler-catheterization study. Circulation 102(15), 1788-1794. doi: 10.1161/01.cir.102.15.1788.

Osman, N.F., McVeigh, E.R., and Prince, J.L. (2000). Imaging heart motion using harmonic phase MRI. IEEE Trans Med Imaging 19(3), 186-202. doi: 10.1109/42.845177.

Penicka, M., Linkova, H., Lang, O., Fojt, R., Kocka, V., Vanderheyden, M., et al. (2009). Predictors of improvement of unrepaired moderate ischemic mitral regurgitation in patients undergoing elective isolated coronary artery bypass graft surgery. Circulation 120(15), 1474-1481. doi: 10.1161/CIRCULATIONAHA.108.842104.

Ryf, S., Spiegel, M.A., Gerber, M., and Boesiger, P. (2002). Myocardial tagging with 3D-CSPAMM. J Magn Reson Imaging 16(3), 320-325. doi: 10.1002/jmri.10145.

von Knobelsdorff-Brenkenhoff, F., Pilz, G., and Schulz-Menger, J. (2017). Representation of cardiovascular magnetic resonance in the AHA / ACC guidelines. J Cardiovasc Magn Reson 19(1), 70. doi: 10.1186/s12968-017-0385-Z.

Walker, J.C., Ratcliffe, M.B., Zhang, P., Wallace, A.W., Fata, B., Hsu, E.W., et al. (2005). MRIbased finite-element analysis of left ventricular aneurysm. Am J Physiol Heart Circ Physiol 289(2), H692-700. doi: 10.1152/ajpheart.01226.2004.

Wang, D., Kirby, R.M., Macleod, R.S., and Johnson, C.R. (2013). Inverse Electrocardiographic Source Localization of Ischemia: An Optimization Framework and Finite Element Solution. $J$ Comput Phys 250, 403-424. doi: 10.1016/j.jcp.2013.05.027.

Wenk, J.F., Sun, K., Zhang, Z., Soleimani, M., Ge, L., Saloner, D., et al. (2011). Regional left ventricular myocardial contractility and stress in a finite element model of posterobasal myocardial infarction. J Biomech Eng 133(4), 044501. doi: 10.1115/1.4003438.

Wijns, W., Vatner, S.F., and Camici, P.G. (1998). Hibernating myocardium. N Engl J Med 339(3), 173-181. doi: 10.1056/NEJM199807163390307. 


\section{Figure Legends}

411 Figure 1. Flowchart of the proposed method. Note that the raw data are in yellow, the derived pressure

412 in blue, the MRI-derived geometry and strains in green, and the FE modeling process in gray.

413 Figure 2. Examples for 4 sequences of images collected in this study: (A) short axis Cine-MRI with

414 LV epicardial and endocardial and RV endocardial contours; (B) a stress perfusion image with arrows

415 indicating the ischemic region; (C) a late gadolinium enhancement (LGE/infarct) image with red 416 arrows indicating the infarcted region, and (D) a short axis tagged image.

417 Figure 3. (A) RV endocardial surfaces (triangle mesh), LV epicardial surface (hexahedral mesh), and 418 the septum (in green) determined using a ray-casting method; (B) the final FE model built with 17 419 sectors with the septal sectors in green.

420 Figure 4. Comparison of the average of $E_{C C}$ in the synthetic test of Patient 1 (A) and Patient 2 (B).

421 Figure 5. (A) Optimization history of the overall objective function, and (B) surface plot of mean422 squared-error of strain respect to $\operatorname{Tmax}_{H}$ and $\alpha$.

423 Figure 6. The convergence of the parameter (lines in blue represent the upper and lower bounds) for 424 (A) $\operatorname{Tmax}_{H}$ for healthy myocardium, and (B) ischemia effect, $\alpha$, on the LV myocardial contractility, 425 with each parameter resulted in a precise final converged optimum.

426 Figure 7. Comparison of the average of $E_{C C}$ with the standard error of the mean in all three cases for 427 (A) all sectors included and (B) only sectors with LGE $<3$.

428 Figure 8. Comparison of the average of $E_{C C}$ of Patient 1 at baseline and after virtual revascularization. 429 
430 Table 1. Patient-specific LV and RV pressures

\begin{tabular}{|c|c|c|c|}
\hline & Patient 1 & Patient 2 & Volunteer \\
\hline LV EDP [mmHg] & 20 & 10 & 10 \\
\hline LV ESP [mmHg] & 140 & 140 & 120 \\
\hline RV EDP [mmHg] & 8 & 8 & 8 \\
\hline RV ESP [mmHg] & 28 & 40 & 25 \\
\hline
\end{tabular}

431

432 
433 Table 2. Patient-specific LGE, SP, and wall motion scores.

\begin{tabular}{|c|c|c|c|c|c|c|}
\hline & \multicolumn{3}{|c|}{ Patient 1} & \multicolumn{3}{|c|}{ Patient 2} \\
\hline Segment & LGE score & $\begin{array}{c}\text { Stress } \\
\text { perfusion } \\
\text { score } \\
\end{array}$ & $\begin{array}{l}\text { Wall motion } \\
\text { score }\end{array}$ & LGE score & $\begin{array}{c}\text { Stress } \\
\text { perfusion } \\
\text { score } \\
\end{array}$ & $\begin{array}{l}\text { Wall motion } \\
\text { score }\end{array}$ \\
\hline 1 & 0 & 0 & 0 & 0 & 0 & 0 \\
\hline 2 & 0 & 0 & 0 & 0 & 0 & 0 \\
\hline 3 & 0 & 2 & 1 & 0 & 0 & 0 \\
\hline 4 & 0 & 3 & 3 & 0 & 1 & 0 \\
\hline 5 & 3 & 3 & 4 & 4 & 1 & 2 \\
\hline 6 & 0 & 1 & 1 & 0 & 0 & 0 \\
\hline 7 & 1 & 2 & 3 & 0 & 2 & 0 \\
\hline 8 & 0 & 1 & 0 & 0 & 0 & 0 \\
\hline 9 & 0 & 1 & 0 & 0 & 0 & 0 \\
\hline 10 & 1 & 2 & 3 & 1 & 0 & 1 \\
\hline 11 & 3 & 2 & 3 & 4 & 0 & 3 \\
\hline 12 & 1 & 1 & 1 & 0 & 0 & 1 \\
\hline 13 & 1 & 3 & 3 & 1 & 1 & 0 \\
\hline 14 & 0 & 1 & 0 & 0 & 0 & 0 \\
\hline 15 & 0 & 2 & 3 & 0 & 0 & 1 \\
\hline 16 & 0 & 0 & 1 & 3 & 0 & 3 \\
\hline 17 & 0 & 0 & 3 & 0 & 0 & 0 \\
\hline
\end{tabular}

434 
436 Table 3. Mesh convergence study.

\begin{tabular}{|c|c|c|c|c|c|}
\hline & $\begin{array}{c}\text { Elements } \\
\text { numbers }\end{array}$ & $\mathrm{C}_{H}$ & $\operatorname{Tmax}_{\boldsymbol{H}}(\mathrm{kPa})$ & $\alpha$ & $\begin{array}{l}\text { Calculation } \\
\text { time }\left(\mathrm{h}^{*} \mathrm{cpu}\right)\end{array}$ \\
\hline \multirow{4}{*}{ Patient 1} & 2700 & 2.07 & 322.7 & 0.08 & 9.6 \\
\hline & 4176 & 2.07 & 338.9 & 0.10 & 15.5 \\
\hline & 7952 & 1.92 & 336.8 & 0.11 & 35.0 \\
\hline & 10752 & 1.92 & 330.7 & 0.11 & 50.0 \\
\hline \multirow{4}{*}{ Patient 2} & 2700 & 0.095 & 405.3 & 0.0 & 15.5 \\
\hline & 4176 & 0.095 & 414.8 & 0.0 & 25.0 \\
\hline & 7952 & 0.094 & 401.4 & 0.0 & 80.0 \\
\hline & 10752 & 0.090 & 408.6 & 0.0 & 112.0 \\
\hline Volunteer & 7952 & 0.0011 & 259.4 & N/A & 345.9 \\
\hline
\end{tabular}


438 Table 4. The synthetic test

\begin{tabular}{|c|c|c|c|c|}
\hline \multirow{2}{*}{ ID } & \multicolumn{2}{|c|}{ Input } & \multicolumn{2}{c|}{ Optimized } \\
\cline { 2 - 5 } & $\operatorname{Tmax}_{H}(\mathrm{kPa})$ & $\alpha$ & $\operatorname{Tmax}_{H}(\mathrm{kPa})$ & $\alpha$ \\
\hline Patient 1 & 336.8 & 0.0 & 337.6 & 0.0 \\
\hline Patient 1 & 336.8 & 1.0 & 332.2 & 0.0 \\
\hline Patient 2 & 401.4 & 0.0 & 401.5 & 1.0 \\
\hline Patient 2 & 401.4 & 1.0 & 401.0 & 0 \\
\hline
\end{tabular}


440 Table 5. MRI-based in vivo volumes and the FE-predicted volumes after optimization

\begin{tabular}{|c|c|c|c|c|}
\hline \multirow{2}{*}{ ID } & \multicolumn{2}{|c|}{ MRI-based } & \multicolumn{2}{c|}{ FE-predicted } \\
\cline { 2 - 5 } & EDV $(\mathrm{ml})$ & ESV $(\mathrm{ml})$ & EDV (ml) & ESV (ml) \\
\hline Patient 1 & 160.9 & 111.0 & 160.9 & 108.3 \\
\hline Patient 2 & 94.9 & 52.2 & 94.9 & 51.3 \\
\hline Volunteer & 118.7 & 49.7 & 118.7 & 48.6 \\
\hline
\end{tabular}




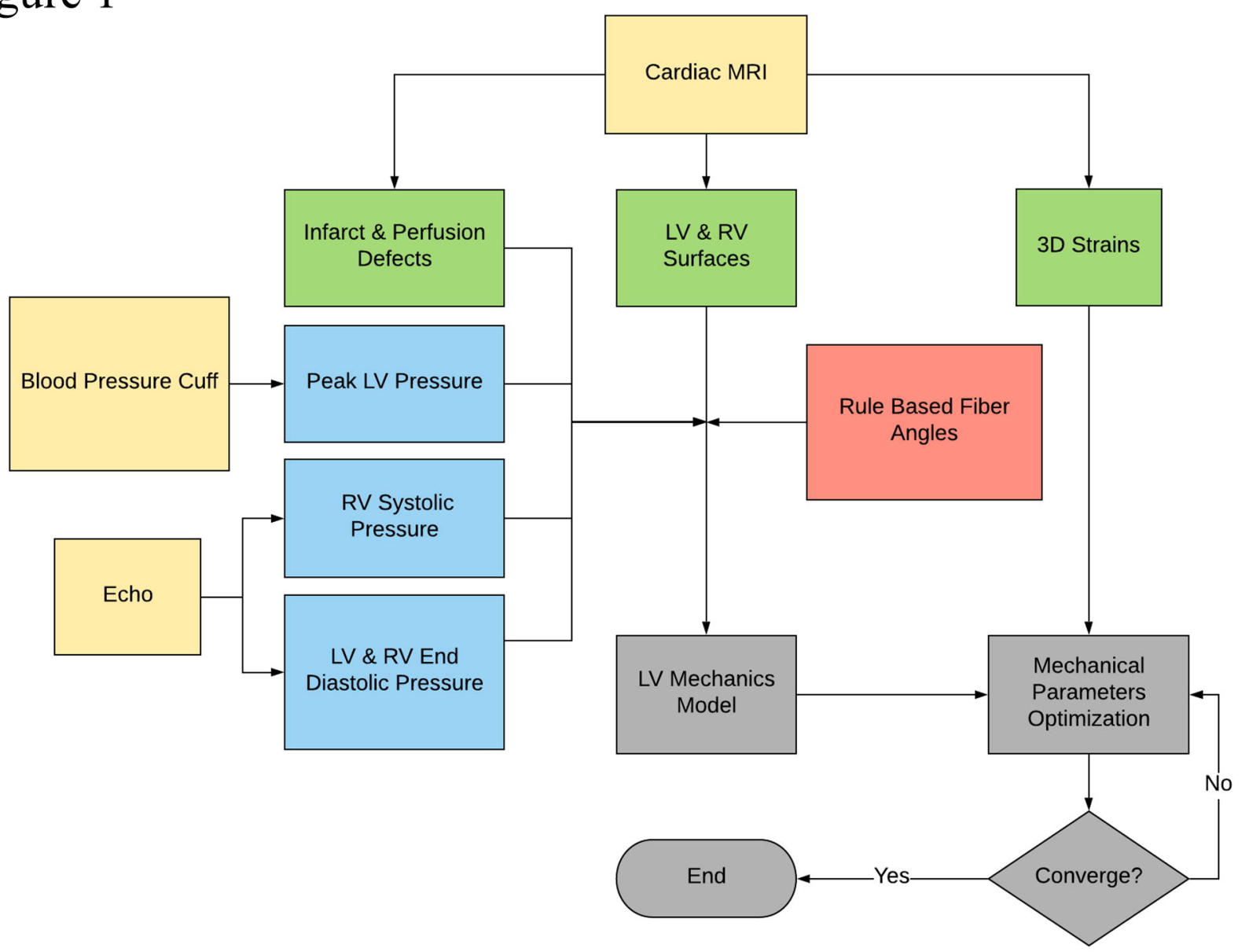


Figure 2
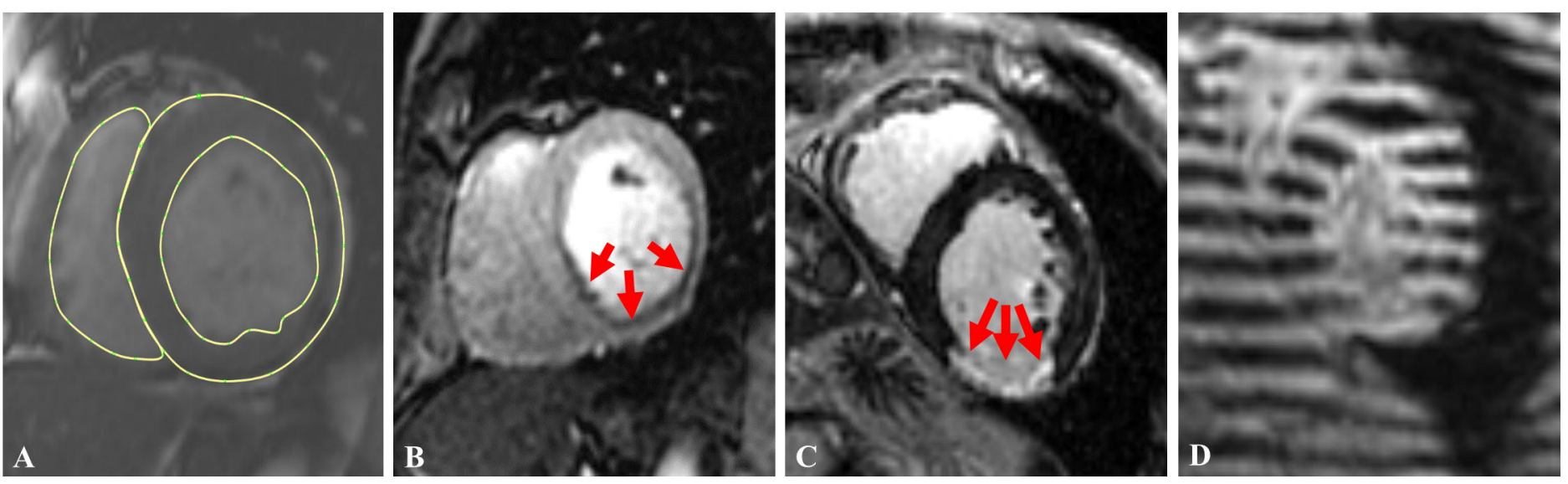
Figure 3

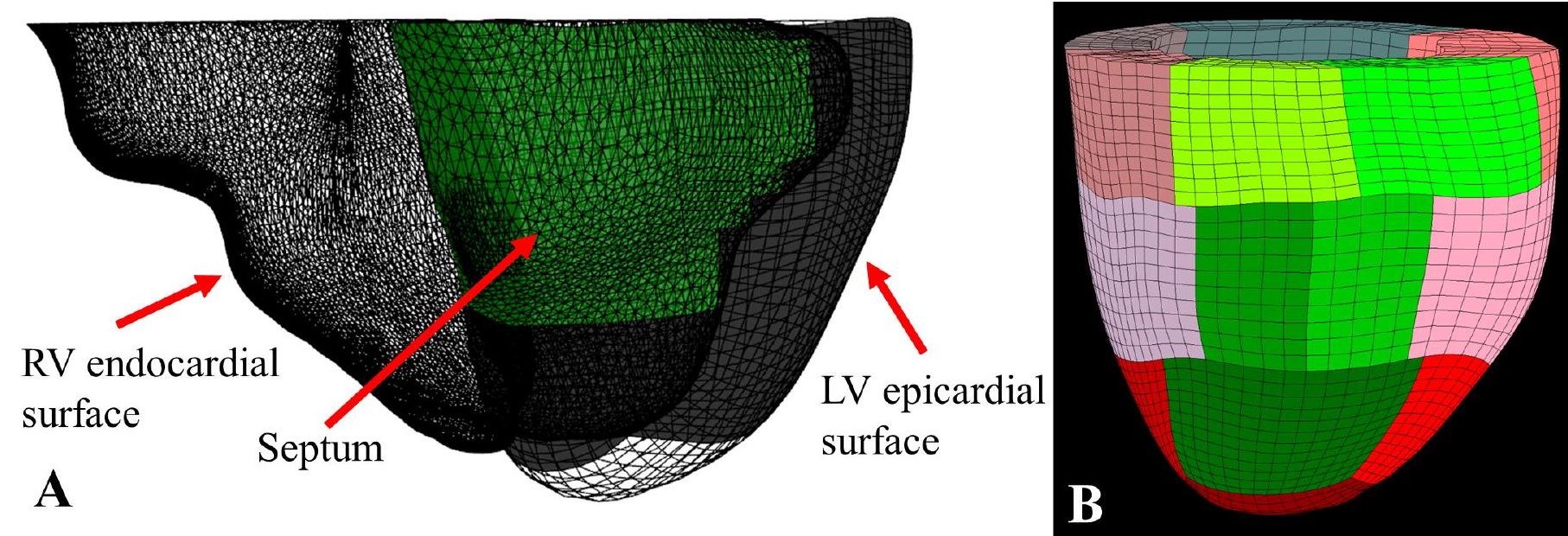


Figure 4

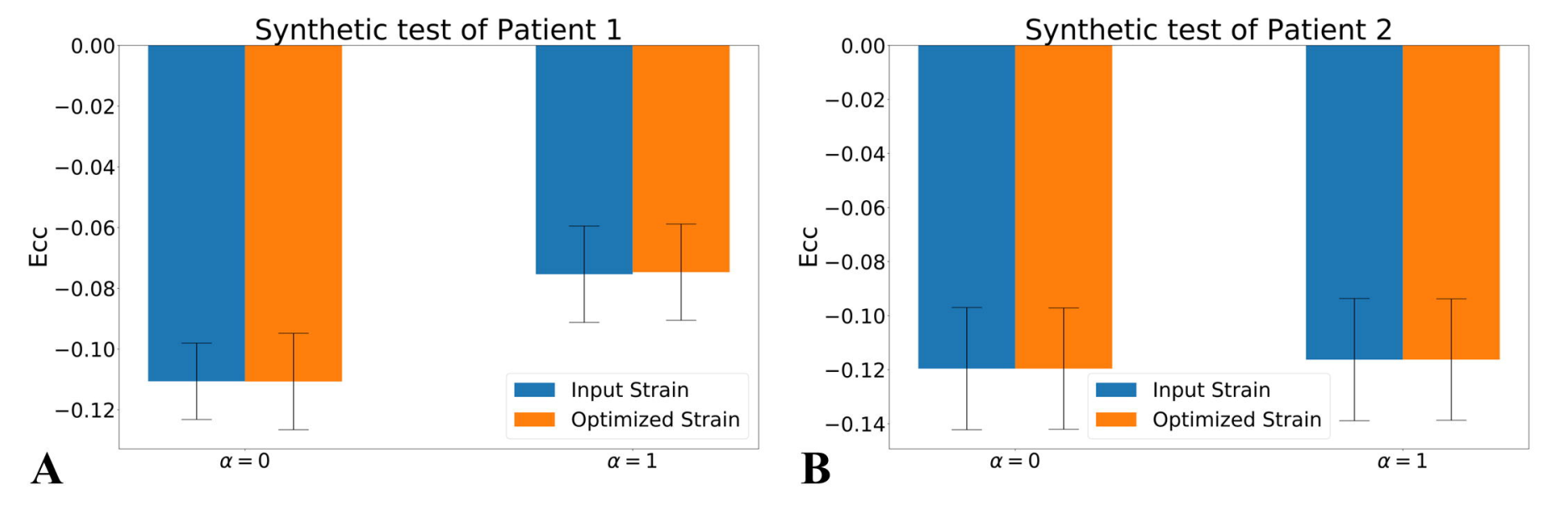


Figure 6
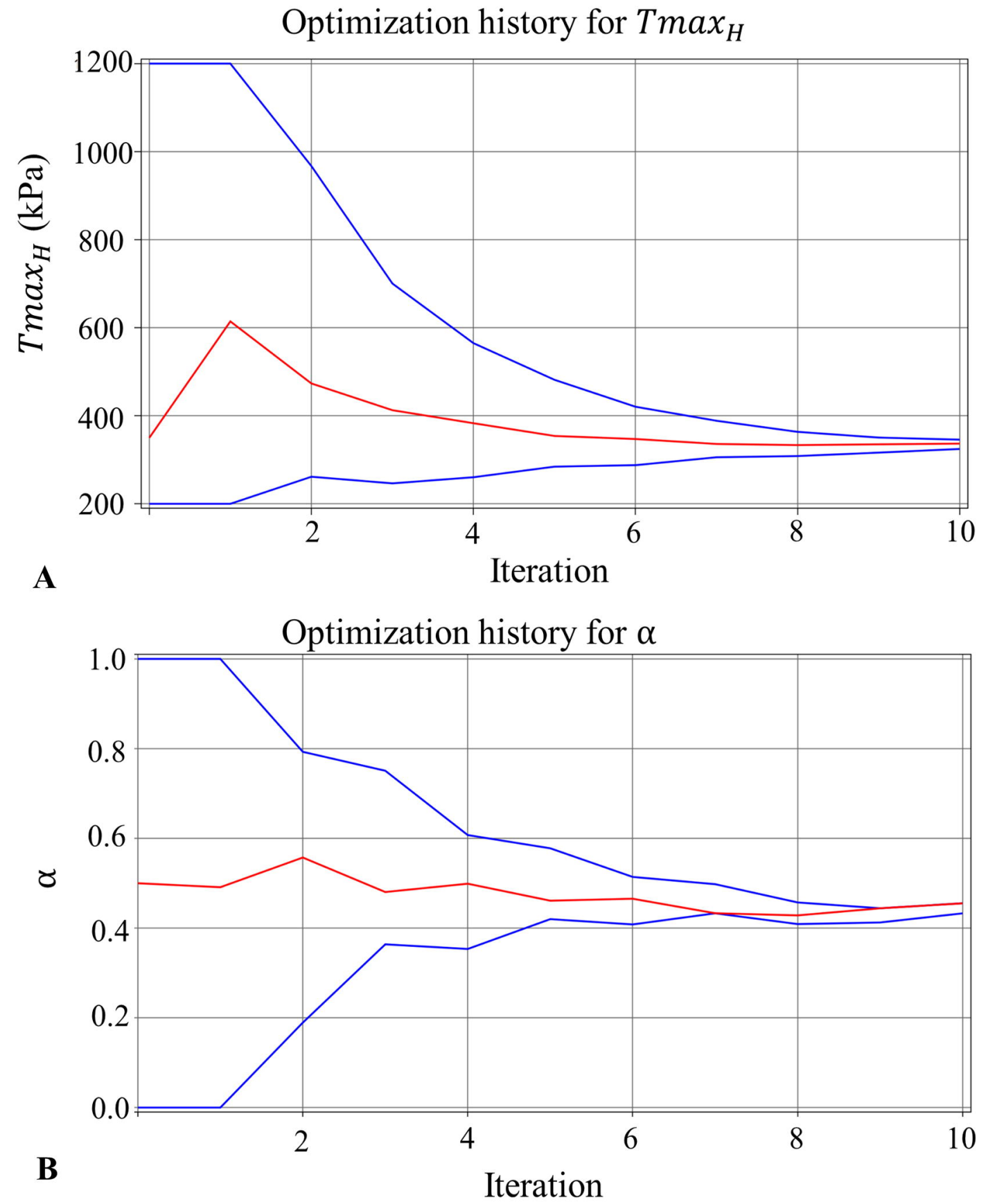
Figure 7
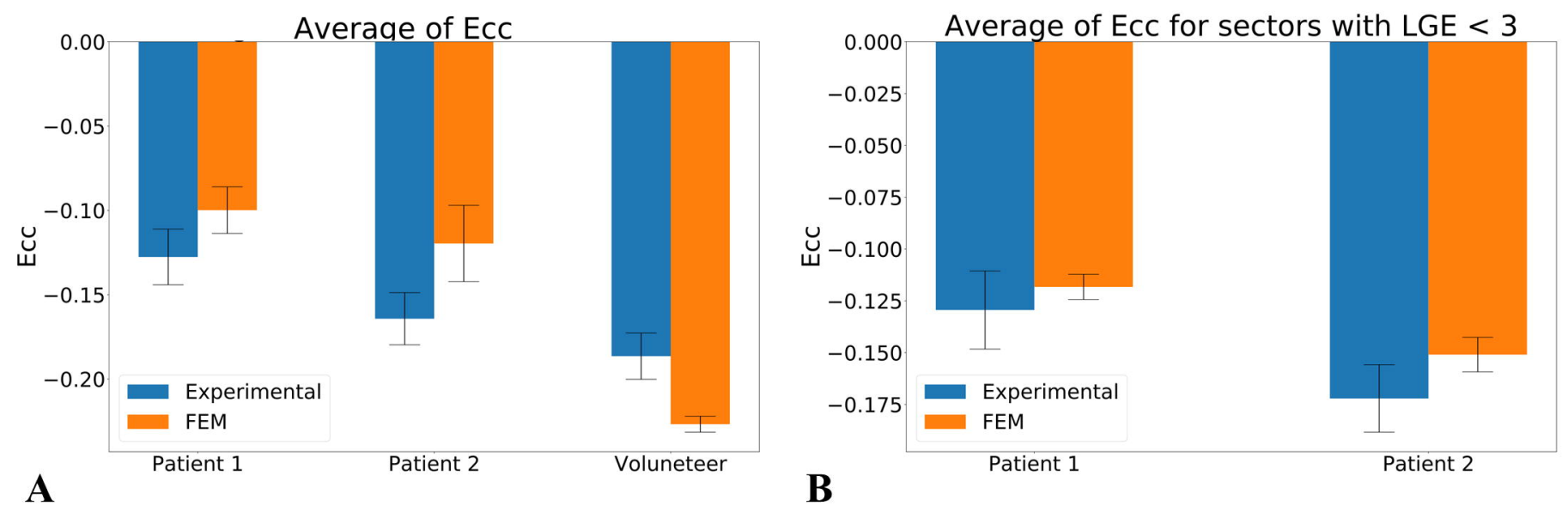

A

B 
Figure 8

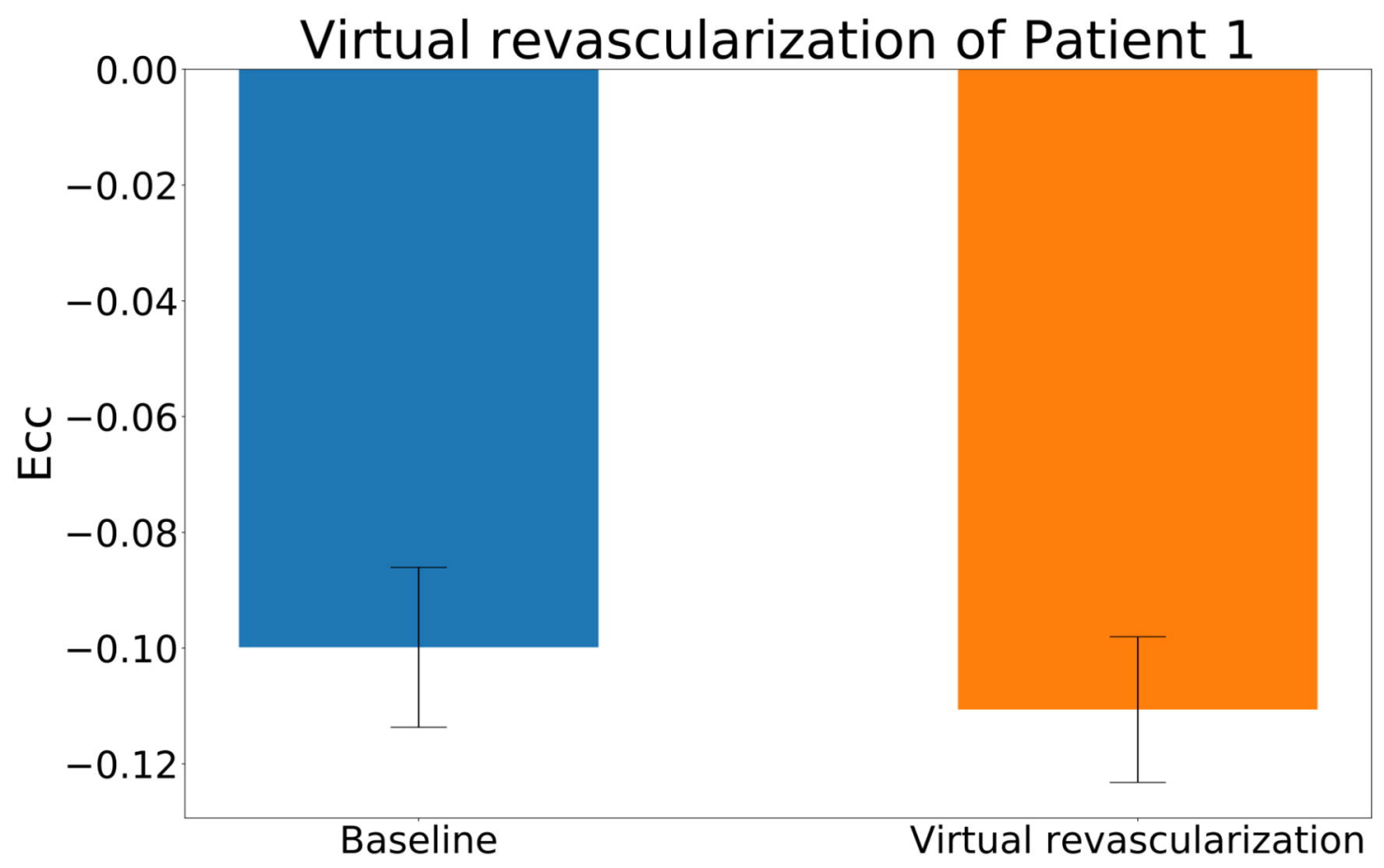

